\title{
Improving Exercise Adherence and Physical Measures in English-Speaking Latina Women
}

\author{
Lorena Martin $^{1}$ • Joseph F. Signorile ${ }^{2}$ Barbara E. Kahn ${ }^{3}$ - Andrew W. Perkins ${ }^{4}$. \\ Soyeon $\mathrm{Ahn}^{2} \cdot$ Arlette C. Perry ${ }^{2}$
}

Received: 13 November 2014 / Revised: 23 February 2015 / Accepted: 27 February 2015 / Published online: 24 March 2015

(C) W. Montague Cobb-NMA Health Institute 2015

\begin{abstract}
Background Epidemiological data have established that lack of physical activity increases risk factors for chronic diseases. Data also suggests that physical activity participation is lowest in minority women, particularly Latinas, and that the nature of the exercise and attitudes toward exercise may influence exercise adherence. The purpose of this study was to determine the effect of hypertrophy training (HT) or power training (PT) used concomitantly with evaluative conditioning (EC) or neutral conditioning (NC) on exercise adherence as well as in physical and psychosocial variables in Latina women. EC is a behavioral method using paired stimuli to develop and strengthen new associations in memory.

Methods Two hundred thirty-one English-speaking Latina women (mean $\pm \mathrm{SD}$, age $36.8 \pm 15.9$ years) were randomly
\end{abstract}

Lorena Martin holds a Ph.D., University of California San Diego.

Joseph F. Signorile holds a Ph.D., University of Miami.

Barbara E. Kahn holds a PhD, The Wharton School of the University of Pennsylvania.

Andrew W. Perkins holds a Ph.D., Washington State University.

Soyeon Ahn holds a Ph.D., University of Miami.

Arlette C. Perry holds a Ph.D., University of Miami.

Lorena Martin

lomartin@ucsd.edu

1 Department of Family Medicine and Public Health, University of California San Diego, La Jolla, CA 92093, USA

2 University of Miami, Coral Gables, FL 33124, USA

3 The Wharton School of the University of Pennsylvania, Philadelphia, PA 19104, USA

4 Washington State University, Pullman, WA 99164, USA assigned to standard HT or high-speed circuit PT and then further stratified to receive EC or NC.

Results One hundred forty-two Latina women completed preand posttesting. For HT, EC produced significantly greater exercise time across the training period than NC. HT and PT both significantly improved all physical, functional, and psychosocial variables, with exception of leg extension and usual gait speed $(p<0.05)$.

Conclusion EC can positively impact exercise adherence during HT in Latina women; while body composition, neuromuscular, and functional performances can be increased using HT and PT independent of psychological conditioning.

Keywords Latina women · Health disparities $\cdot$ Psychology Resistance training $\cdot$ Circuit training $\cdot$ Physical activity

\section{Introduction}

Epidemiological data have established that a lack of physical activity increases risk factors for chronic diseases and is reportedly responsible for up to 250,000 deaths per year, most of which are preventable [1]. Unfortunately, less than one half (49.1\%) of US adults meet the Centers for Disease Control and Prevention (CDC)/American College of Sports Medicine (ACSM) physical activity recommendations [2]. Despite being aware of the importance of regular exercise, the National Health Interview Survey data for 2010 reported that $33 \%$ of adults in this country do not engage in any leisure time physical activity and $49 \%$ do not meet aerobic or resistance training federal guidelines for physical activity [3].

Physical activity is lowest among minority groups; particularly Latina women [4], who by 2050 are expected to 
represent $42 \%$ of the US population [5]. According to the US Department of Health and Services Office of Women's Health, six out of 10 Latinas are not physically active, adding to the burden of high chronic disease rates that can be prevented by regular exercise [6]. Recently, the federal guidelines for physical activity added resistance training to the recommendations as a result of the positive gains from strength training. The benefits of resistance training include improved cardiovascular fitness, visceral fat, total body composition, lipid profiles, blood pressure, and psychosocial variables such as self-esteem and body image. Since Latinas are reported to have low adherence rates to other types of physical activity, it would seem vital to examine the effects of resistance training in this population, which may possibly result in different adherence rates. It would be novel to assess whether resistance training results in greater physical activity participation in Latinas as to our knowledge, adherence to resistance training has yet to be examined in a Latina population.

Even among persons who have made a conscious decision to engage in exercise programs, poor compliance and high dropout rates are a central issue [7]. The average attrition rate for physical activity and exercise programs has been estimated to be $55 \%$, with the majority of dropouts occurring within the first four weeks of training $[8,9]$. The poor adherence rates observed in these supervised exercise training programs further exacerbate the current low levels of physical activity participation among Latina women.

The benefits of exercise and physical activity on psychosocial variables are well known, particularly, the construct of self-esteem, the evaluation of one's self-worth [10]. Moreover, a relationship between body image and self-esteem has been clearly demonstrated, although some results are conflicting [11]. Furthermore, research has shown women to be more likely to have body image issues, usually being more dissatisfied with their appearance and therefore less motivated to perform exercise [11]. Although a number of factors, such as cultural norms, socioeconomic status, and social support systems, may affect levels of physical activity, research indicates that the individual's attitude concerning the importance of exercise is one of the most important determining factors [12]. In a study by Eyler et al. [12], attitudes toward physical activity were an important contributing factor affecting adherence levels in Latina women. Lack of belief in ability to perform a certain behavior, in this case exercise, better known as exercise self-efficacy along with low body esteem have also been reported as contributing factors hindering physical activity participation [13].

Behavioral scientists have examined various theoretical models such as the Learning Theory, Decision-Making Theory, the Ecological Model Theory, and Social Cognitive Theory (SCT) and how they can be implemented effectively with the objective of getting people to start and/or maintain an exercise program and lead a healthy lifestyle. Social cognitive theory states that knowledge, perceived self-efficacy, and outcome expectations are major factors that influence our behaviors [14]. Based on the nature of this experiment, SCT was the basis for the assessment of pre- and postexercise self-efficacy [14]. Although SCT has been applied to aerobic exercise training and has resulted in positive findings, it has not, to our knowledge, yet been applied to resistance training in Latinas. Given the fact that resistance training has recently been added to the exercise guidelines proposed by ACSM and the Surgeon General's Report on Physical Activity and Health it is important to examine the influence of SCT on resistance training [15]. The nature of a resistance training intervention is commonly described using four factors: load, movement speed, modality, and exercise patterns. The loading continuum is defined as the maximum number of repetitions $(\mathrm{RM})$ an individual can complete with a specific load before stopping. However, a one-repetition maximum, the maximum amount of weight that can be lifted in one repetition, is usually obtained prior to estimating a specified RM training regimen. For example, a 3-5RM (maximum amount of weight that can be lifted between three to five times) range is typically used to increase strength, an 8-12RM range for hypertrophy, and a 2028RM range for endurance [16]. When power is the primary goal, increases in speed are superimposed over these basic loading patterns. The balance between load and velocity, often termed optimal load, depends upon specific factors such as training goals, joint biomechanics, and training status [17]. The modality used may vary from free weights, through selectorized machines and even bands or rubber tubing. Finally, the typical patterns involve either single or multiple sets, or circuits.

Currently, the two loading patterns that dominate the literature on fitness and health-based interventions in non-athletic populations are hypertrophy training (HT) and power training (PT). Although there is not a linear relationship between changes in strength and muscle cross-sectional area, HT is often considered synonymous with strength training in the literature [18]. While HT at 70-80 \% 1RM (8-12RM) has been used to increase muscle cross-sectional area and strength, lower weights (40-60\% 1RM) have typically been used to achieve the optimal PT load associated with the greatest product of load and velocity in untrained individuals, usually resulting in greater improvements in body composition and muscular power [17]. Although both forms of training have been effectively used to enhance health-related outcomes, no single study, to our knowledge, has examined the effects of HT and PT on body composition, neuromuscular, functional, and psychosocial variables. Given the positive impact of resistance training it would be interesting to see if evaluative conditioning (EC) could further enhance adherence to resistance training, particularly in women. EC has been shown to decrease or increase behaviors through the use of image pairing [19].

Supportive evidence has been presented on the effectiveness of using aversive images to communicate potential health 
consequences of unhealthy behaviors, as well as appetitive images to reinforce positive behaviors. This innovative modality, EC, may be a means to modify unhealthy behaviors and increase healthy behaviors. EC is a method by which stimuli is paired with other stimuli with the objective of strengthening or developing new associations [19]. Since this technique has been effectively used to reduce smoking and racial biases, and alter food choices; it may be useful in modifying physical activity behavior, specifically exercise adherence [20,21]. It is not known whether HT or PT combined with EC will result in better adherence rates among Latina women.

The purpose of this study is to determine whether HT or PT used concomitantly with EC can result in better adherence to resistance training programs in Latina women than training coupled with sessions of neutral conditioning (NC). A secondary purpose is to determine whether HT or PT in combination with EC or NC will lead to similar changes in body composition, neuromuscular, functional, or psychosocial variables in Latina women. We hypothesize that the addition of EC to either HT or PT will result in better exercise adherence, and that PT in conjunction with EC will produce greater positive changes in body composition, neuromuscular, functional, or psychosocial variables in the Latina women participating in our study.

\section{Methods}

Subjects Latina participants were recruited from the local Miami area through advertisements in local magazines, newspapers, university campuses, and radio stations. Eligibility criteria included: English-speaking Latinas between the ages of 18-69; willingness to attend EC or NC sessions conducted once every 3 weeks; the ability to attend resistance training sessions one or more times per week, and being physically inactive within the last 6 months. Exclusion criteria were: any known cardiovascular, metabolic, neuromuscular, or pulmonary disease, pregnancy, any clinical mental disorders, or any other medical conditions. Six subjects were excluded for medical issues. All the participants started the trainings at the same time. The study consisted of a total of 12 weeks that included a 2 -week lead acclimatization phase, pre- and posttesting. A total of 290 participants were recruited of which 231 were eligible and randomized to one of four groups; HTEC, PTEC, HTNC, and PTNC. A total of 145 participants completed all pretests and posttests (Fig. 1).

Design A randomized, 10-week intervention was conducted in the Laboratories of Clinical and Applied Physiology and

\section{CONSORT FLOW DIAGRAM}

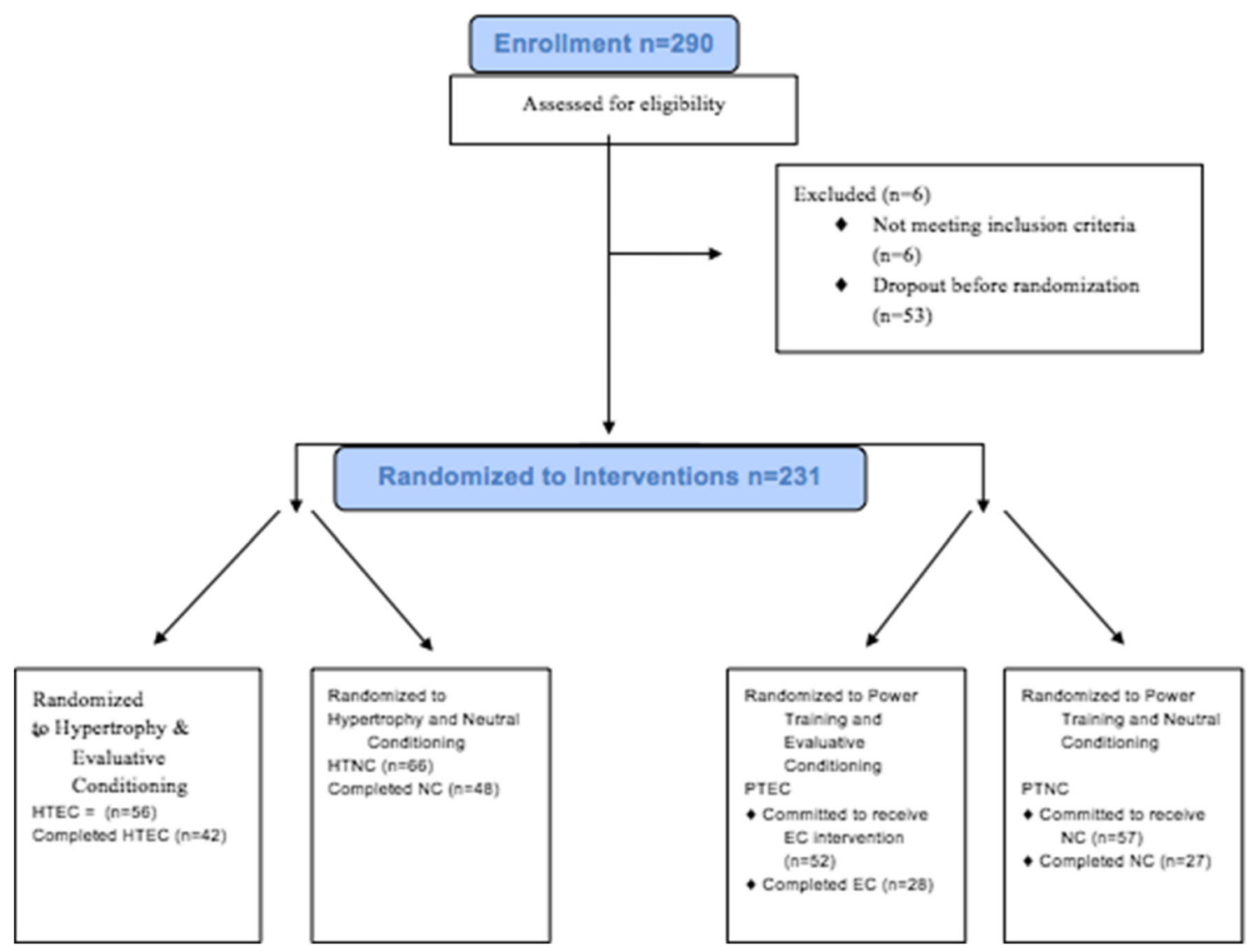

Fig. 1 Consort flow chart depicting recruitment and adherence rates among participants 
Neuromuscular Research and Active Aging of the University of Miami to determine the effects of EC together with resistance training on exercise adherence, body composition, neuromuscular, functional, and psychosocial variables. The laboratories were made available for resistance training MondayFriday, 6 am-10 pm, and Saturdays from 9-1 pm to accommodate the number of women participating in the study. Participants were randomized to one of the four groups after meeting the inclusion criteria. Block randomization was used to control for age and weight. Investigators were blinded to the subjects' assignments to either EC or NC; however, they were aware of the type of resistance training participants received. Only the head investigator and her assistant knew which subjects received EC or NC.

Prior to testing, all personnel were required to attend a mandatory two-weekend workshop during which they practiced testing and training procedures. Following this workshop, inter-rater reliability ranged from 0.93 to 0.98 for body composition, neuromuscular, and functional variables.

Hypertrophy Training HT sessions consisted of three sets of eight repetitions performed for nine exercises (biceps curl, leg extension, leg curls, shoulder press, leg press, back row, chest press, lat pull-down, and triceps dips) at $80 \%$ of participants' 1RM. Concentric and eccentric portions of the lift averaged between 1 and $2 \mathrm{~s}$. Two-minute rest intervals were provided between sets.

Power Training PT sessions consisted of three sets of 13 repetitions using aforementioned exercises mentioned performed at $50 \%$ of participants' $1 \mathrm{RM}$ with 2-min rest intervals between sets. The concentric portion of each exercise was performed as explosively as possible concentrating on maximal load-dependent velocity, while the eccentric portion was performed at the same speed used for HT training. Power training is also commonly recognized as high-speed circuit training. The number of repetitions, 8 and 13, for HT and $\mathrm{PT}$, respectively, were chosen to provide an equivalent volume of work during each protocol.

Evaluative Conditioning On scheduled EC training days, participants were escorted to the Behavioral Research Laboratory prior to their resistance training sessions to complete EC. Instructions were given in English only. Participants were instructed to sit in a chair in front of a desktop computer, place on earphones, and input their ID numbers when prompted by the instructions displayed on the computer screen. Once their IDs were recorded, the assigned EC was automatically initiated. During the EC sessions, participants were presented with positive words paired with images of healthy behaviors or neutral words paired with images unrelated to healthy behaviors. They were then instructed to click on the spacebar whenever they were presented with the target images (Fig. 2)
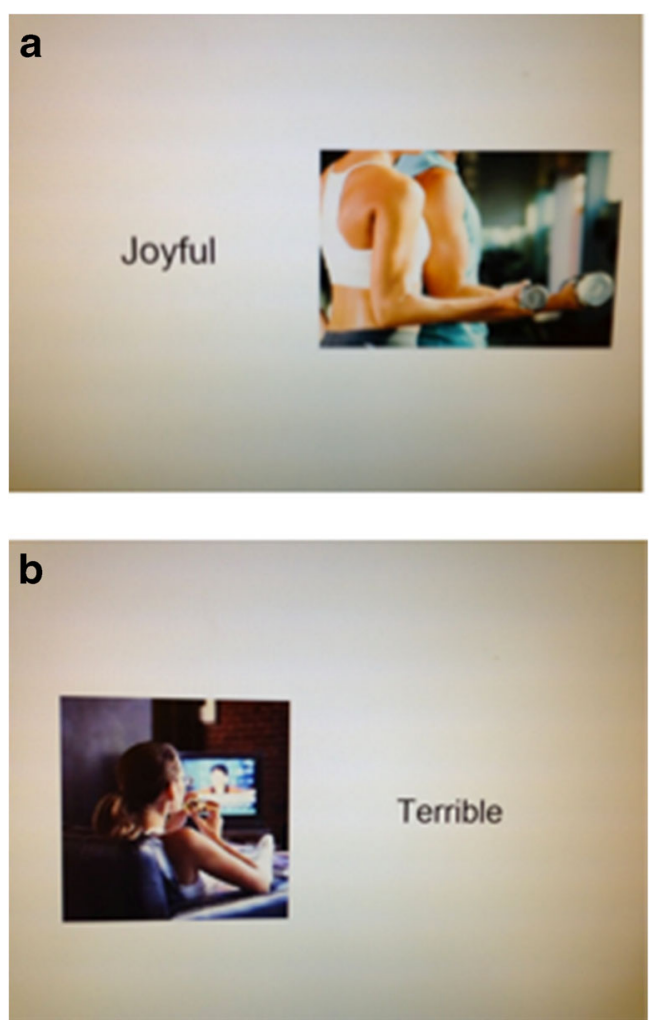

Fig. 2 Evaluate conditioning demonstrating pairing of positive (joyful) and negative (terrible) targets paired with images representing healthy (a) and unhealthy (b) behavior

associated with "healthy behaviors". Three conditioning trials were provided approximately every three weeks throughout the 10 -week intervention.

Neutral Conditioning For NC, the same procedure as EC was followed, with the exception that the $\mathrm{NC}$ group received a dummy task containing unrelated target images paired with neutral words unrelated to the focus variables.

\section{Testing Procedures}

Exercise Adherence Exercise adherence was assessed using the amount of time spent training quantified in minutes. For each training session the subject logged in and out using a designated laboratory computer. Only training performed in the laboratory was used to calculate adherence levels.

Body Composition Body composition variables included body mass index (BMI), percent body fat, lean body mass, and waist and hip circumferences. BMI was calculated as weight in kilograms divided by height in meters squared. Percent body fat and lean body mass was evaluated using bioelectrical impedance (Inbody 520; GE Healthcare; Waukesha, WI). The Inbody 520 is an established measure of adiposity and lean body mass [22]. Waist and hip circumferences were 
taken using a spring-loaded tape (Gay Mills, WI). Waist circumference was measured mid-way between the lowest rib and the iliac crest, while hip circumference was measured at the level of the greater trochanters. Trained staff assessed body composition, where inter-rater reliability ranged was 0.93 for body composition variables.

Neuromuscular Performance A 1RM testing protocol was used to assess the maximal force production for the Cybex plate-loaded machines and the Keiser pneumatic leg press. For Keiser lower body power testing, a modification of the protocol by Thomas et al. [23] was used in which participants performed three repetitions as rapidly as possible at $50 \%$ of their 1RM for leg press.

Functional Capacity Functional variables included the $30 \mathrm{~s}$ Arm Curl (30sAC), $30 \mathrm{~s}$ Chair Stand (30sCS), usual $\left(\mathrm{GS}_{\mathrm{usl}}\right)$ and maximum gait speeds $\left(\mathrm{GS}_{\max }\right)$, reactive balance (RB; Proprio 5000; Perry Dynamics Inc., Decatur, IL), and the single leg stand test (SLS).

Psychosocial Self-Reports Psychosocial measures included the Rosenberg Self-Esteem Scale (RSE), the Exercise Selfefficacy scale (ESES), and the Body Esteem scale (BES). The RSE is a widely used measure of global self-esteem with 10 items that has high internal consistency of 0.77 , with a reported Coefficient of Reproducibility of 0.90 or more [24]. The ESES is a questionnaire in which participants rate confidence in their ability to perform exercise, regardless of the situation, with 18 items on a scale from 0-100. Bandura's ESES scale has a single factor structure with high internal consistency (0.95) [14]. The BES is a 35-item questionnaire in which participants indicate how they feel about each body part and function of their bodies using a five-point Likert scale [25]. Internal consistency has been evaluated with values ranging from 0.82 to 0.94 .

\section{Statistical Analysis}

All data were analyzed using SPSS 19 with standard statistical methods being used to calculate means, standard deviations, confidence intervals, skewness, and kurtosis on all dependent variables. There were no baseline differences $(p=0.580)$ in body composition, neuromuscular, functional, and psychosocial variables with the exception of exercise self-efficacy (see below), which was controlled for statistically. A $2 \times 2$ ANOVA was used to examine the effects of two types of resistance training (HT or PT) and conditioning (EC or NC) on exercise adherence quantified using the total number of minutes at the training facility. Four $2 \times 2$ MANOVAs were conducted to evaluate changes in body composition (BMI, percent body fat, lean body mass, and waist and hip circumference), neuromuscular performance (upper and lower body strength and lower body power), functional capacity ( $30 \mathrm{~s}$ chair stand, $30 \mathrm{~s}$ arm curl, usual gait speed and maximal gait speed tests, Proprio 5000, and the single leg stand), and psychosocial factors (self-esteem, body esteem-physical attractiveness, weight concern, and physical condition) following resistance training (HT, PT). An ANCOVA was used to control for baseline differences in exercise self-efficacy. For significant main effects, post hoc analyses were performed. For significant interactions, analyses of simple effects were performed. An alpha level of 0.05 was used for all analyses with post hoc Bonferroni correction used for multiple comparisons.

\section{Results}

Subject characteristics including age, weight, height, and BMI are presented in Table 1. As indicated by BMI, subjects were on average, overweight, but not obese. The age of subjects ranged from 18-69 with a mean age of 36.8 years. Of the initial 284 women available to participate in the study, 231 were pre-tested and randomly assigned to HT and PT groups. A total of 145 participants completed both the pre- and posttests. Of these subjects, 90 subjects in the HT group $(\mathrm{EC}=42$; $\mathrm{NC}=48$ ) completed posttesting and 55 subjects in the PT group $(\mathrm{EC}=28 ; \mathrm{NC}=27)$ completed posttesting.

Our principal findings were that both training modality and EC had an impact on exercise adherence in Latina women. There was a significant training $\times$ conditioning interaction for exercise adherence (Fig. 3). For HT, the EC group demonstrated a significantly longer time spent training than the NC group $\left(F(1,184)=11.874, p<0.001, \eta_{\mathrm{p}}{ }^{2}=0.061\right)$. For PT, there was no significant difference between $\mathrm{EC}$ and $\mathrm{NC}$ groups in time spent training; however, both groups spent significantly more minutes training than the $\mathrm{NC}$ group engaged in $\mathrm{HT}$.

Table 2 presents the pretest and posttest means \pm SD for body composition, neuromuscular, functional, and psychosocial variables. For BMI, percent body fat, waist circumference and hip circumference, a main effect showing improvement in these variables was seen with training $(p<0.001)$. For neuromuscular performance, all exercises, with the exception of the leg extension, showed significant increases in strength following training $(p<0.05)$. For the Keiser pneumatic leg press, a significant increase in strength was observed following training $(p=0.048)$. Training also resulted in significant
Table 1 Subject characteristics $(N=145)$. Values are mean $\pm \mathrm{SD}$

\begin{tabular}{lll}
\hline Characteristics & Mean & SD \\
\hline Age (years) & 36.8 & 15.82 \\
Weight $(\mathrm{kg})$ & 69.5 & 14.8 \\
Height $(\mathrm{m})$ & 1.59 & 0.21 \\
BMI $\left(\mathrm{kg} \cdot \mathrm{m}^{2}\right)$ & 27.46 & 7.12 \\
\hline
\end{tabular}


Fig. 3 Exercise adherence to hypertrophy and power training utilizing evaluate or neutral conditioning. Values represent means + SEM. *Significantly greater than neutral conditioning during hypertrophy training, $p<0.01$. **Significantly greater than neutral conditioning during hypertrophy training, $p<0.001$

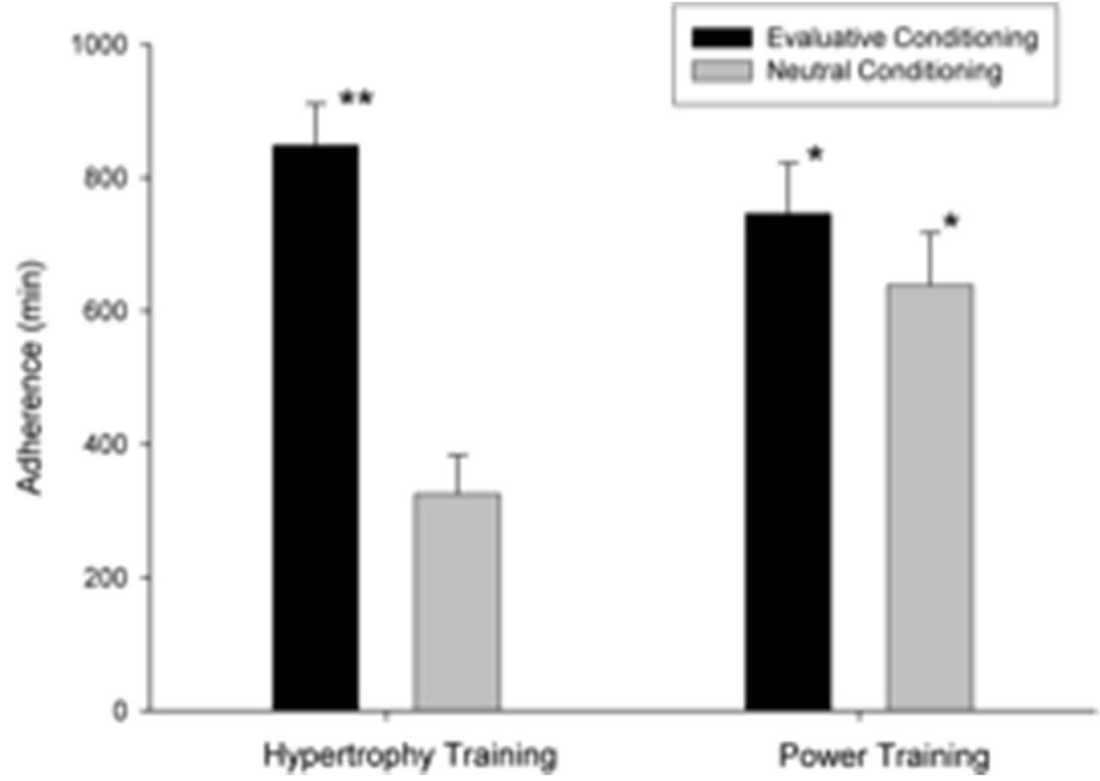

improvements in all functional variables $(p<0.05)$ with the exception of usual gait speed. Significant increases were also evidenced for psychosocial variables; self-esteem and body esteem variables by resistance training $(p<0.05)$.

A significant time $\times$ condition interaction was found for lean body mass $\left(F(1,76)=5.138, p<0.05, \eta_{\mathrm{p}}{ }^{2}=0.063\right)$ (Fig. 4). Pairwise comparisons showed that for NC, lean body mass declined across the training period, while no significant difference in LBM was seen for the EC group. Additionally, a significant time $\times$ condition interaction was also observed for exercise self-efficacy ( $p<0.05$; Fig. 5). For the EC condition, exercise self-efficacy significantly increased from pretest to posttest $\left(F_{1,52}=5.146, P<0.05, \eta_{\mathrm{p}}{ }^{2}=0.065\right)$.

\section{Discussion}

Our hypothesis that EC would increase exercise adherence in Latina women was supported when EC was combined with HT, which employed fewer repetitions and heavier loads than PT. This may have been due to the fact that EC served to offset the negative effect on adherence resulting from the higher perceived intensity associated with the higher loads used during HT. Ekkekakis [26] found that as exercise intensity increased, there was a decrease in pleasure, and as a consequence, a negative impact on exercise adherence.

In contrast, PT produced similar adherence rates for both EC and NC. The same explanation may account for the limited effectiveness of EC with PT. The lower loads associated with PT likely engendered lower levels of perceived exertion and consequently greater adherence independent of EC. As White et al. [27] noted in their review of factors related to physical activity adherence in women, low-intensity exercise may result in greater adherence than high-intensity programs, especially during the early stages of training. This may explain why adherence for the PT group was independent of EC or NC. Our results are also supported by the findings of the Sedentary Women Exercise Adherence Trial (S.W.E.A.T.) Study, which showed that moderate intensity exercise produced a higher level of exercise training retention than vigorous exercise [28].

The present study confirms earlier findings that both HT and PT can positively impact BMI, percent body fat, and waist circumference. Although studies have shown that declines in BMI can be found following resistance training, results were equivocal showing increases and no change in this variable $[29,30]$. These divergent results are not difficult to explain since, as Prentice and Jabb [31] have stated, BMI is merely a surrogate measure of body composition and an ineffectual measure of body fat in individuals who have increased muscle mass due to training. Our results demonstrating a decrease in waist circumference and percent body fat with both HT and PT also reflect those reported in previous studies using circuit and standard resistance training programs [32].

Waist circumference is an excellent indicator of central obesity. The reduced waist circumference levels seen in this study may have important health implications, particularly in Latina women, who suffer from higher rates of diabetes and hypertension than Caucasian women [33]. To our knowledge, the reduction in hip circumference observed in Latina women in this study has not previously been reported; however, these results concur with the decreases found in BMI and waist circumference. The significant decrease in LBM for the NC group may be explained in part by the fact that a lesser amount of time was spent training by participants in this group compared to those in the EC groups.

Our findings that significant gains in upper and lower body strength were seen for both HT and PT groups are consistent 
Table 2 Pretest and posttest values for body composition, neuromuscular, functional, and psychosocial variables following resistance exercise adjusted for multiple comparisons using Bonferroni correction

\begin{tabular}{|c|c|c|c|c|}
\hline & Pre & Post & $p$ & Effect sizes \\
\hline \multicolumn{5}{|l|}{ Body composition } \\
\hline Body mass index $\left(\mathrm{kg} \mathrm{m}^{-2}\right)$ & $27.31 \pm 6.29$ & $25.48 \pm 5.51$ & $0.0001 * *$ & 0.31 \\
\hline Body Fat (\%) & $34.58 \pm 9.33$ & $32.99 \pm 9.42$ & $0.0001 * *$ & 0.33 \\
\hline Waist circumference (cm) & $32.70 \pm 6.17$ & $30.34 \pm 5.84$ & $0.0001 * *$ & 0.47 \\
\hline Hip circumference (cm) & $41.21 \pm 5.65$ & $39.06 \pm 5.14$ & $0.0001 * *$ & 0.34 \\
\hline \multicolumn{5}{|l|}{ Neuromuscular } \\
\hline Keiser leg press strength $(\mathrm{N})$ & $1040 \pm 282.65$ & $1188 \pm 464.99$ & $p=0.048$ & ns \\
\hline Keiser leg press power (W) & $77.59 \pm 13.61$ & $82.23 \pm 11.18$ & $p=0.125$ & ns \\
\hline Leg extension (kg) & $47.5 \pm 18.3$ & $51.6 \pm 12.8$ & $p=0.231$ & ns \\
\hline Biceps curl strength (kg) & $12.3 \pm 3.9$ & $15.0 \pm 3.5$ & $p=0.006^{* *}$ & 0.21 \\
\hline Leg curl strength (kg) & $37.4 \pm 8.8$ & $45.4 \pm 14.5$ & $p=0.000 * *$ & 0.36 \\
\hline Shoulder press strength $(\mathrm{kg})$ & $21.0 \pm 5.4$ & $24.2 \pm 5.3$ & $p=0.000 * *$ & 0.34 \\
\hline Leg press strength $(\mathrm{kg})$ & $82.0 \pm 27.1$ & $94.1 \pm 28.0$ & $p=0.003 * *$ & 0.24 \\
\hline Back row $(\mathrm{kg})$ & $39.6 \pm 7.7$ & $44.2 \pm 9.1$ & $p=0.000 * *$ & 0.46 \\
\hline Chest press (kg) & $34.9 \pm 10.0$ & $43.5 \pm 12.8$ & $p=0.0001^{* *}$ & 0.58 \\
\hline Lat pull-down (kg) & $23.7 \pm 7.3$ & $28.4 \pm 7.2$ & $p=0.0001^{* *}$ & 0.57 \\
\hline Assisted triceps dips (kg) & $52.6 \pm 23.9$ & $37.4 \pm 21.7$ & $p=0.0001^{* *}$ & 0.50 \\
\hline \multicolumn{5}{|l|}{ Functionality } \\
\hline $30 \mathrm{~s}$ arm curl (repetitions) & $17.09 \pm 3.75$ & $23.89 \pm 4.59$ & $p=0.0001^{* *}$ & 0.73 \\
\hline $30 \mathrm{~s}$ chair stand (repetitions) & $19.26 \pm 5.21$ & $26.15 \pm 6.05$ & $p=0.0001^{* *}$ & 0.57 \\
\hline Usual gait speed (s) & $7.99 \pm 1.32$ & $8.12 \pm 1.32$ & $p=0.457$ & ns \\
\hline Maximal gait speed (s) & $5.10 \pm 0.842$ & $5.32 \pm 0.785$ & $p=0.021^{\mathrm{a}}$ & ns \\
\hline Proprio 5000 DMA Score & $631.68 \pm 143.47$ & $588.57 \pm 162.05$ & $p=0.001 * *$ & 0.16 \\
\hline Single leg stand (s) & $89.41 \pm 37.65$ & $103.58 \pm 29.76$ & $p=0.002 * *$ & 0.14 \\
\hline \multicolumn{5}{|l|}{ Psychosocial } \\
\hline Self-esteem & $7.13 \pm 1.41$ & $7.57 \pm 1.37$ & $p=0.011^{*}$ & 0.11 \\
\hline \multicolumn{5}{|l|}{ Body esteem } \\
\hline Sexual attraction & $48.95 \pm 7.87$ & $52.56 \pm 8.86$ & $p=0.014^{*}$ & 0.10 \\
\hline Weight concern & $26.77 \pm 7.82$ & $34.63 \pm 9.62$ & $p=0.000^{* *}$ & 0.32 \\
\hline Physical condition & $29.55 \pm 6.68$ & $36.13 \pm 7.05$ & $p=0.000^{* *}$ & 0.47 \\
\hline
\end{tabular}

${ }^{*} p<0.05 ; * * p<0.01$

${ }^{\text {a }}$ Not significant after using Bonferroni correction with previous results. In younger women, Cullinen and Caldwell [34] reported significant increases in elbow and knee flexion and extension following 12 weeks of moderate intensity resistance training performed twice per week using a combination of free weight and selectorized machine exercises. Holviala et al. [35] examined the effects of 21 weeks of progressive resistance training and demonstrated significant increases in bilateral leg extension 1RM, and maximum isometric force and average force developed within the first $500 \mathrm{~ms}$ of an isometric bilateral leg extension. Reflective of our results, showing significant increases in maximal gait speed, single leg stand, and Proprio 5000 DMA score, the improvements in strength seen in the study by Holviala et al. [35] translated into improvements in both gait speed and dynamic balance.
The significant increase in pneumatic strength, and not power, for the HT group has been reported elsewhere by Signorile et al. [36]. The lack of difference in leg power between the PT (pretest: $78.3 \pm 11.1 \mathrm{~W}$; posttest: $83.9 \pm 8.9 \mathrm{~W}$ ) and HT (pretest: $77.3 \pm 14.8 \mathrm{~W}$; posttest: $81.5 \pm 12.4 \mathrm{~W}$ ) protocols was unexpected and causal factors are not apparent. One reason for the lack of distinction between the two groups may have been the reluctance of subjects to provide maximal efforts during the power-testing component of the Keiser evaluation; however, this was not evident during testing. A second possibility may have been the use of selectorized machines for PT. Preliminary results from our laboratories indicate that resistance is not maintained throughout the entire range of motion when these machines are employed during high-speed training. 
Fig. 4 Pre- and posttest lean body mass scores for evaluate and neutral conditioning. Values represent means + SEM.

* Significantly lower than pretest scores, $p<0.05$

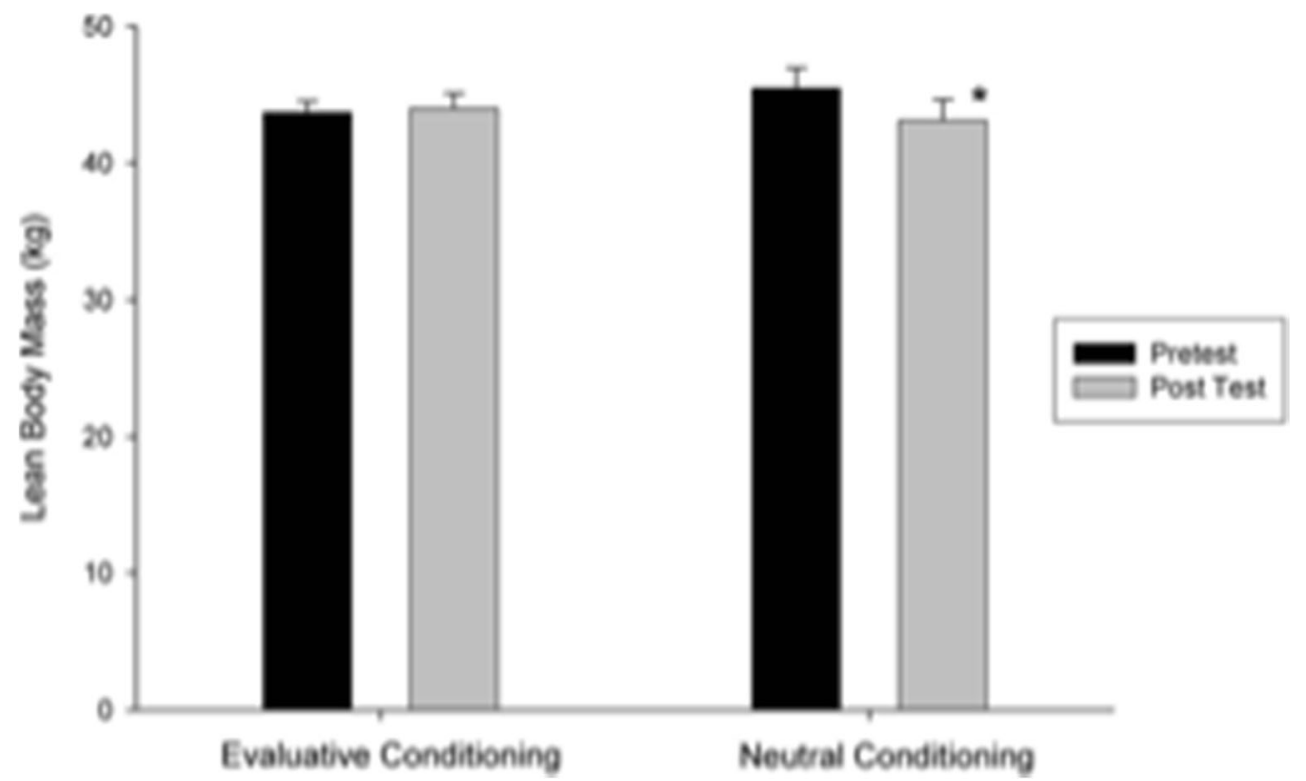

Our data, showing significant improvements in all functional variables with the exception of usual gait speed, is supported by results of previous studies incorporating both younger and older women. In their systematic review of progressive resistance training and older adults using 3674 participants across 62 trials, Latham et al. [37] reported increases in chair rise and gait speed due to training. Additionally, standard resistance training has been shown to produce increases in timed up-and-go, chair stand, and gait speed in active 60year old women that were training-frequency dependent. Our improvements in the 30-s arm curl, 30-s chair stand, single leg stand, maximal gait speed test, and Proprio tests also reflect gains in power and balance demonstrated in other studies [37]. Independent of the type of training, both HT and PT groups evidenced significant improvements on tests of functionality. This finding may be related to the wider age range of the Latina group, 18-69, thereby diluting results and potential increases typically observed in elderly men and women using PT.

Our results showing that self-esteem and body esteem improved following training and mirror those reported by other researchers. In a study examining the influence of a 20 -week
Fig. 5 Pre- and posttest exercise self-efficacy utilizing evaluative and neutral conditioning. Values represent means + SEM.

* Significantly greater than pretest, $p<0.05$

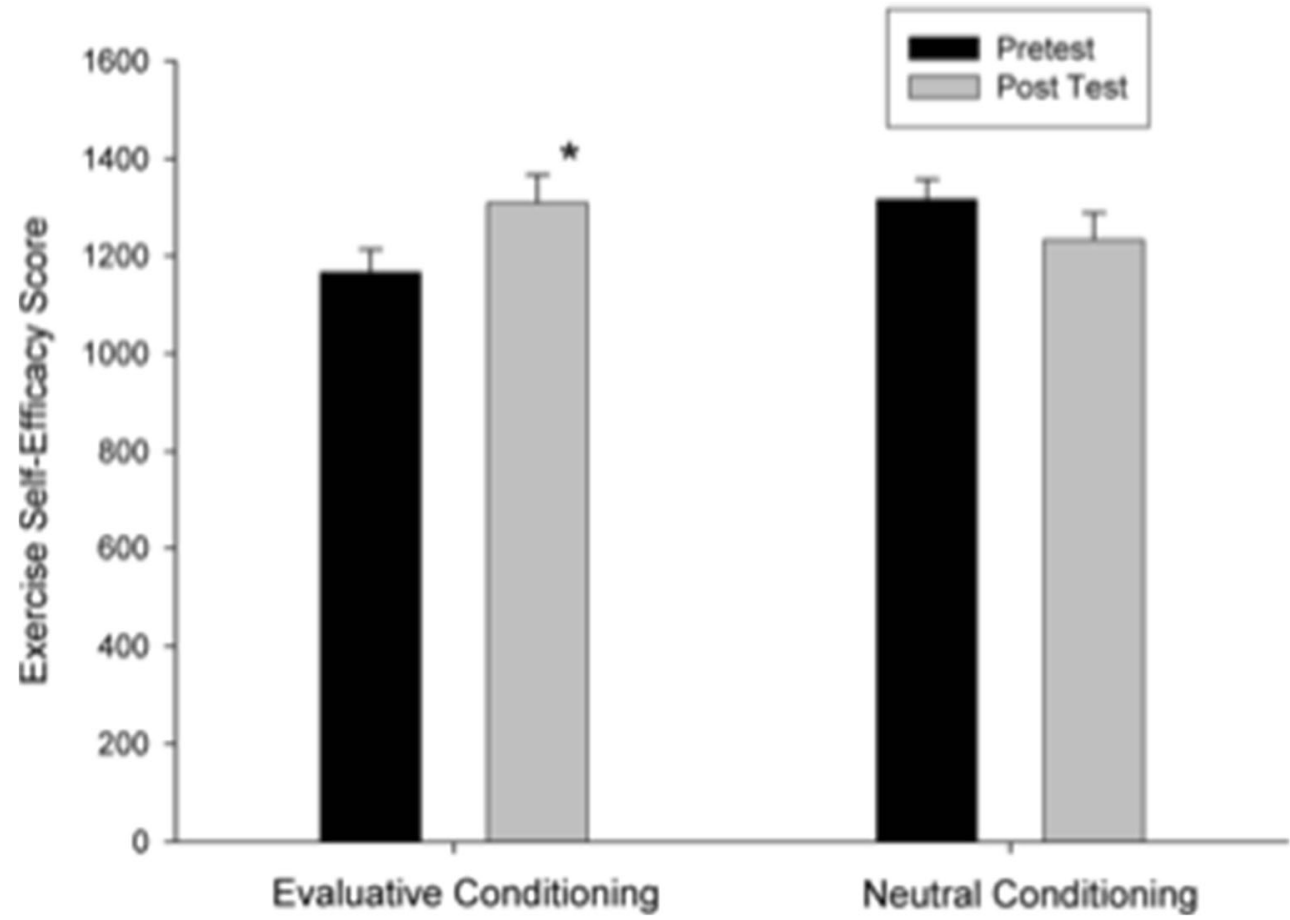


exercise program on self-esteem and exercise self-efficacy in middle-aged adults, McAuley et al. [38] reported significant improvements in global self-esteem and physical self-worth. Although the effect of EC on exercise self-efficacy has not been previously examined; however, a study by Hollands et al. [39] examined the impact of a single bout of EC on food choices, concerns about diet, health consequences, coherence between representations and recommended behavior, and selfefficacy. In contrast to our findings, these researchers reported no impact of EC on any of these variables except implicit attitudes. The difference between our findings and those of Hollands et al. as they relate to self-efficacy, may be attributed to the length of our intervention, a 10 -week intervention, while Hollands et al. examined immediate responses to a single bout of EC [39].

There are several limitations that may have impacted the results of our study. First, women were required to attend at least one session per week and only those attending at least $50 \%$ of those sessions were included in the analyses [40, 41]. This may have affected the amount of time subjects spent training. Second, a substantial percentage of women dropped out of the HT and PT groups since they did not want to commit to receiving any psychological conditioning. Third, no attempt was made to control diet, which may have influenced some of the outcome variables. Furthermore, since the women were required to be fluent in the English language, it is important to acknowledge that this may have excluded those who are not well acculturated, or that experience significant language and cultural barriers, suggesting that future studies include interpreters in order to include a more diverse sample. Lastly, acceptability of type of exercise is another factor that we strongly suggest future similar studies to assess pre- and posttraining. Our observations related to acceptability of strength training was that although they may have had reservations at the start, participants who continually engaged in the training sessions seemed to have developed a positive association with strength training by the end of the study.

Our study sample size $(n=231)$ was larger than those commonly seen in other training studies examining exercise behaviors, which have ranged from 20-60 participants. Although women were asked to attend at least one session per week, participants were provided the opportunity to train up to 6 days per week. Furthermore, participants received three sessions of EC in comparison to previous studies in which only one session was provided. The three sessions were equally distributed across the 10-week training program in order to maximize the impact of EC while minimizing the possibility of a learning effect. Moreover, we chose to employ the suggestion of previous EC studies to include a neutral conditioning (NC) group [42]. Finally, a subtle EC paradigm was incorporated in which neutral and irrelevant images were presented to minimize awareness of our purpose and reduce social desirability and demand characteristics.

\section{Conclusion}

The results of the current study suggest that EC can positively impact exercise adherence in Latina women by associating healthy behaviors with positive words and images. The impact of EC on exercise adherence, however, appears to be modulated by the nature of the exercise used during the intervention, with EC having a greater impact on higher intensity HT versus lower intensity PT. Our findings further support the fact that body composition, neuromuscular performance, functional capacity, and psychosocial variables can be increased using either HT or PT without the use of EC. Further research in the domain of physical activity and exercise adherence using EC is suggested to clarify its value in reducing attrition rates using other exercise paradigms.

Acknowledgements and Funding Source Funding for the current study was provided by a University of Miami Barbara Marks/Katy Dean Research Award and a Transformative Research Grant from the Association for Consumer Research.

Compliance with Ethical Standards There are no potential conflicts of interest and all testing and training procedures were approved by the University of Miami Institutional Review Board and participant provided written informed consents prior to participating in the study.

Conflict of Interest Author Lorena Martin, Author Joseph F. Signorile, Author Barbara E. Kahn, Author Andrew W. Perkins, Author Soyeon Ahn, and Author Arlette C. Perry declare that they have no conflict of interest.

Informed Consent All procedures followed were in accordance with the ethical standards of the responsible committee on human experimentation (institutional and national) and with the Helsinki Declaration of 1975, as revised in 2000 (5). Informed consent was obtained from all patients for being included in the study.

\section{References}

1. Pate RR, Pratt M, Blair SN, Haskell WL, Macera CA, Bouchard C, et al. Physical activity and public health. JAMA. 1995;273(5):4027.

2. Garber CCE. Quantity and quality of exercise for developing and maintaining cardiorespiratory, musculoskeletal, and neuromotor fitness in apparently healthy adults guidance for prescribing exercise. Med Sci Sports Exerc. 2011;43(7):1334-59.

3. Centers for Disease Control and Prevention. Summary health statistics for U.S. adults. NHIS; 2010.10:252.

4. Cromwell SL, Berg JA. Lifelong physical activity patterns of sedentary Mexican American women. Geriatr Nurs. 2006;27(4):209 13.

5. Vincent GK, Velkoff VA. The next four decades: the older population in the United States: 2010 to 2050 (No. 1138). US Department of Commerce, Economics and Statistics Administration, US Census Bureau; 2010.

6. Booth FW, Gordon SE, Carlson CJ, Hamilton MT. Waging war on modern chronic diseases: primary prevention through exercise biology. J Appl Physiol. 2000;88(2):774-87. 
7. Marcus BH, Williams DM, Dubbert PM, Sallis JF, King AC, Yancey AK, et al. Physical activity intervention studies what we know and what we need to know: a scientific statement from the American Heart Association Council on Nutrition, Physical Activity, and Metabolism (subcommittee on physical activity); Council on Cardiovascular Disease in the Young; and the Interdisciplinary Working Group on Quality of Care and Outcomes Research. Circulation. 2006;114(24):2739-52.

8. Jacobsen DJ. Adherence and attrition with intermittent and continuous exercise in overweight women. Int J Sports Med. 2003;24(6): 459-64.

9. Dishman R, Sallis J, Orenstein D. The determinants of physical activity and exercise. Public Health Rep. 1985;100(2):158-71.

10. McAuley EE. Exercise and self-esteem in middle-aged adults: multidimensional relationships and physical fitness and self-efficacy influences. J Behav Med. 1997;20(1):67-83.

11. Furnham A, Badmin N, Sneade I. Body image dissatisfaction: gender differences in eating attitudes, self-esteem, and reasons for exercise. J Psychol. 2002;136(6):581-96.

12. Eyler AA, Vest JR, Sanderson B, Wilbur J, Matson-Koffman D, Evenson KR, et al. Environmental, policy, and cultural factors related to physical activity in a diverse sample of women: the Women's cardiovascular health network project introduction and methodology. Women Health. 2002;36(2):1-15.

13. Schwarzer R, Renner B. Social-cognitive predictors of health behavior: action self-efficacy and coping self-efficacy. Health Psychol. 2000;19(5):487-95.

14. Bandura A. Social cognitive theory: an agentic perspective. Annu Rev Psychol. 2001;52(1):1-26.

15. United States. Department of Health, \& Human Services. (1996). Physical activity and health: a report of the Surgeon General. DIANE Publishing.

16. Campos GE, Luecke TJ, Wendeln HK, Toma K, Hagerman FC, Murray TF, et al. Muscular adaptations in response to three different resistance-training regimens: specificity of repetition maximum training zones. Eur J Appl Physiol. 2002;88(1):50-60.

17. Kawamori N, Haff GG. The optimal training load for the development of muscular power. J Strength Cond Res. 2004;18(3):675-84.

18. Goodpaster BH, Park SW, Harris TB, Kritchevsky SB, Nevitt M, Schwartz AV, et al. The loss of skeletal muscle strength, mass, and quality in older adults: the health, aging and body composition study. J Gerontol A Biol Sci Med Sci. 2006;61(10):1059-64.

19. De Houwer J, Thomas S, Baeyens F. Associative learning of likes and dislikes: a review of 25 years of research on human evaluative conditioning. Psychol Bull. 2001;127(6):853-69.

20. Baeyens F, Eelen P, Crombez G, Van den Bergh O. Human evaluative conditioning: acquisition trials, presentation schedule, evaluative style and contingency awareness. Behav Res Ther. 1992;30(2): 133-42.

21. Olson MA, Fazio RH. Reducing automatically activated racial prejudice through implicit evaluative conditioning. Personal Soc Psychol Bull. 2006;32(4):421-33.

22. Pietrobelli A, Rubiano F, St-Onge M, Heymsfield S. New bioimpedance analysis system: improved phenotyping with whole-body analysis. Eur J Clin Nutr. 2004;58(11):1479-84.

23. Thomas M, Fiatarone MA, Fielding RA. Leg extensor power in young women: functional correlates and relationship to body composition and strength. Med Sci Sports Exerc. 1996;28:1321-6.

24. Pullmann H, Allik J. The Rosenberg Self-Esteem Scale: its dimensionality, stability and personality correlates in Estonian. Personal Individ Differ. 2000;28(4):701-15.

25. Franzoi SL, Shields SA. The Body Esteem Scale: multidimensional structure and sex differences in a college population. J Pers Assess. 1984;48(2):173-8.
26. Ekkekakis P, Hall EE, Petruzzello SJ. The relationship between exercise intensity and affective responses demystified: to crack the 40 -year-old nut, replace the 40-year-old nutcracker! Ann Behav Med. 2008;35(2):136-49.

27. White JL, Ransdell LB, Vener J, Flohr JA. Factors related to physical activity adherence in women: review and suggestions for future research. Women Health. 2005;41(4):123-48.

28. Cox K, Burke V, Gorely T, Beilin L, Puddey I. Controlled comparison of retention and adherence in home-vs center-initiated exercise interventions in women ages 40-65 years: the SWEAT study (Sedentary Women Exercise Adherence Trial). Prev Med. 2003;36(1):17-29.

29. Benton MJ, Kasper MJ, Raab SA, Waggener GT, Swan PD. Shortterm effects of resistance training frequency on body composition and strength in middle-aged women. J Strength Cond Res. 2011;25(11):3142.

30. Tibana RA, Navalta J, Bottaro M, Vieira D, Tajra V, de Oliveira Silva A, et al. Effects of eight weeks of resistance training on the risk factors of metabolic syndrome in overweight/obese women-A Pilot Study. Diabetol MetabSyndr. 2013;5(1):1-8.

31. Prentice AM, Jebb SA. Beyond body mass index. Obes Rev. 2001;2(3):141-7.

32. Dunstan DW, Daly RM, Owen N, Jolley D. High-intensity resistance training improves glycemic control in older patients with type 2 diabetes. Diabetes Care. 2002;25(10):1729-36.

33. Lorig KR, Ritter PL, González VM. Latina chronic disease selfmanagement: a randomized community-based outcome trial. Nurs Res. 2003;52(6):361-9.

34. Cullinen $\mathrm{K}$, Caldwell $\mathrm{M}$. Weight training increases fat-free mass and strength in untrained young women. J Am Diet Assoc. 1998;4:414-8.

35. Holviala JH, Sallinen JM, Kraemer WJ, Alen MJ, Hakkinen KK. Effects of strength training on muscle strength characteristics, functional capabilities, and balance in middle-aged and older women. J Strength Cond Res. 2006;20(2):336-44.

36. Signorile JF, Carmel MP, Czaja S, Asfour S, Morgan RO, Khalil $\mathrm{T}$, et al. Differential increases in average isokinetic power by specific muscle groups of older women due to variations in training and testing. $\mathrm{J}$ Gerontol A Biol Sci Med Sci. 2002;57A(10):M683-90.

37. Latham NK, Bennett DA, Stretton CM, Anderson CS. Systematic review of progressive resistance strength training in older adults. J Gerontol A Biol Sci Med Sci. 2004;59A(1):48-61.

38. McAuley E, Blissmer B, Katula J, Duncan TE, Mihalko SL. Physical activity, self-esteem, and self-efficacy relationships in older adults: a randomized controlled trial. Ann Behav Med. 2000;22(2):131-9.

39. Hollands GJ, Prestwich A, Marteau TM. Using aversive images to enhance healthy food choices and implicit attitudes: an experimental test of evaluative conditioning. Health Psychol. 2011;30(2):195203.

40. Azad NA, Bouchard K, Mayhew A, Carter M, Molnar FJ. Safety and predictors of adherence of a new rehabilitation program for older women with congestive heart failure. J Geriatr Cardiol: JGC. 2012;9(3):243.

41. Acharya SD, Elci OU, Sereika SM, Music E, Styn MA, Turk MW, et al. Adherence to a behavioral weight loss treatment program enhances weight loss and improvements in biomarkers. Patient Prefer Adherence. 2009;3:151.

42. Baeyens F, Field AP, Houwer JD. Associative learning of likes and dislikes: some current controversies and possible ways forward. Cogn Emot. 2005;19(2):161-74. 